Ann. Zootech., I968, 18 (2), I99-205.

\title{
INFLUENCE DE LA SUPPLÉMENTATION EN ANTIBIOTIQUES SUR LA CROISSANCE DES PORCELETS SEVRÉS A 5 SEMAINES : OXYTÉTRAGYCLINE ET OLÉANDOMYCINE
}

\author{
A. AUMAITRE \\ avec la collaboration technique de R. Dexiel \\ Station de Recherches sur l'Élevage des Porcs, \\ Centre national de Recherches sootechniques, 78 -Jouy-en-Josas \\ Institut national de la Recherche agronomique
}

\section{SOMMAIRE}

Deux expériences portant sur l'addition d'oxytétracycline et d'oléandomycine, utilisés seuls ou en association, au régime de sevrage des porcelets à 5 semaines ont été entreprises.

La supplémentation de la ration, à raison de 20 p.p.m. d'oléandomycine ou de 4 o p.p.m. d'oxytétracycline + to p.p.m. d'oléandomycine (Taomycine) entraîne une amélioration de la vitesse de croissance de 29 et $35 \mathrm{p}$. 10o lorsque les animaux sont nourris à volonté. Les quantités d'aliments consommées augmentent dans les mêmes proportions mais l'efficacité alimentaire est identique dans tous les lots.

L'effet de la supplémentation sur la croissance disparaît lorsque les animaux sont nourris en quantités égalisées par rapport au lot témoin; la vitesse de croissance et l'efficacité alimentaire ne sont pas améliorés.

Les antibiotiques ajoutés à l'aliment de sevrage du porcelet semblent agir au niveau de la stimulation de l'appétit des jeunes animaux.

\section{IN'TRODUCTION}

La supplémentation par divers antibiotiques des régimes pour porcelets sous la mère ou des régimes de sevrage précoce est devenue une pratique courante en élevage (BECKER et al., I952; FrançoIs, I962; JoUANDET et al., I964). C'est en effet à cette époque que la stimulation de croissance qu'elle provoque est la plus grande.

L'utilisation continue d'un même antibiotique pose cependant des problèmes liés, d'une part à l'amélioration constante de la qualité et de l'équilibre des rations de sevrage et d'autre part à l'accoutumance possible de la flore microbienne du milieu ou du tube digestif du porcelet. 
Il existe un grand nombre d'antibiotiques efficaces sur la croissance du Porc. Certains cependant comme l'oléandomycine (MAGRUDER et al., I958; FERRANDO, I960; Smith et al., I963) n'ont pas fait l'objet d'observations dans le cas des jeunes animaux en période de sevrage précoce.

Enfin, les associations d'antibiotiques peuvent avoir un effet encore plus favorable sur la croissance lorsque leur action est synergique (RUSSEL, I962). L'oxytétracycline et la pénicilline associées dans la ration du Porc possèdent une telle propriété (BECKER et al., I953). De même l'emploi simultané de l'oxytétracycline et de l'oléandomycine a donné des résultats similaires sur porcelets récemment sevrés (FERRANDo, I960 ; IsAKov, r963; MÜNCHBERG et al., I963; KRÜGER et al., I964).

Cependant, l'effet de ces différentes supplémentations est très variable, les améliorations constatées vont de 5 à $40 \mathrm{p}$. Ioo et leur mécanisme d'action est loin d'être élucidé (FrançOIS, I 962 ; RÉRAT et $a l$., I965). Dans certains cas, la supplémentation augmente l'efficacité alimentaire, dans d'autres, seule la vitesse de croissance est améliorée.

L'effet de la supplémentation d'un régime de sevrage à 5 semaines pour porcelets a été étudié à l'aide de 2 antibiotiques, oxytétracycline et oléandomycine, utilisés seuls ou en association dans le cas d'une alimentation distribuée à volonté ou rationnée.

\section{MATÉRIEL ET MÉTHODES}

\section{Animaux}

Deux expériences ont été réalisées, l'une sur 8 portées ( 64 animaux) en alimentation à volonté, l'autre sur II portées (44 animaux) en alimentation individuelle et rationnée.

Les animaux originaires de notre troupeau expérimental ont été sevrés à 5 semaines, le même jour (expérience r) ou sur une période de 2 mois (expérience 2 ), les mesures ont été effectuées durant 4 semaines après sevrage. Dans chaque expérience, 4 lots étaient constitués :

$$
\begin{aligned}
& \text { I : Témoin } \\
& \text { II : Oxytétracycline } \\
& \text { III : Oléandomycine } \\
& \text { IV : Oxytétracycline + Oléandomycine }
\end{aligned}
$$

La mise en lot est réalisée de la façon suivante :

\section{Expérience 1}

Deux animaux par portée sont affectés ì chacun des 4 lots, suivant le poids et le sexe. On reconstitue un groupe de 8 animaux par traitement, logés ensemble et, à partir des 8 portées, deux répétitions sont réalisées. Les aliments sont placés dans de petits distributeurs automatiques et la consommation est laissée à volonté.

Le poids individuel et les quantités moyennes d'aliment sont notés chaque semaine.

\section{Expérience 2.}

Les animaux sont groupés par 4 au sein d'une même portée selon le poids au sevrage et le sexe. Ils sont maintenus en groupes et alinıentés individuellement 4 fois par jour. La quantité totale d'aliment offerte par semaine est égalisée pour chacun des 4 lots.

Chaque animal est pesé toutes les semaines.

Dans les deux expériences, l'état sanitaire (diarrhée) est soigneusement vérifié chaque jour et rapporté en moyenne par animal pour toute la durée de l'expérience. 


\section{Aliments et supplémentation}

Nous avons utilisé un aliment de sevrage de type classique ${ }^{1}$ ) présenté sous forme de granulé. Cet aliment (lot témoin, I) a été supplémenté de la façon suivante :

- Au taux de roo p.p.m. d'oxytétracycline = lot Oxytétracycline (II)

- Au taux de 20 p.p.m. d'oléandomycine = lot Oléandomycine (III)

- Au taux de $\left\{\begin{array}{l}40 \text { p.p.m. d'oxytétracycline } \\ + \text { Io p.p.m. d'oléandomycine }\end{array}=\right.$ lot "Taomycine " (IV)

Les concentrations réelles d'antibiotiques ont été contrôlées par analyse microbiologique en fin d'expérience ( 2 mois après la fabrication). Ce résultat est présenté au tableau I.

Les calculs sont réalisés par la méthode de comparaison multiple des moyennes.

TABLEAU I

Composition chimique et contrôle de la teneur des aliments expérimentaux en antibiotiques

\begin{tabular}{|c|c|c|c|c|}
\hline Aliment supplémenté & 'Témoin & $\begin{array}{l}\text { Oxytétra- } \\
\text { gycline }\end{array}$ & $\begin{array}{l}\text { Oléando- } \\
\text { mỵcine }\end{array}$ & "Taomycine "( \\
\hline Teneur en jrotéines brutes p. $100^{\prime}$ & 17,2 & 17,2 & 17,3 & 17,2 \\
\hline $\begin{array}{l}\text { Titre theorique en antibiotiques } \\
\qquad(\mathrm{mg} / \mathrm{kg})\end{array}$ & 0 & 100 & 20 & $\begin{array}{l}40 \text { Oxytétracyline } \\
10 \text { Oléandomycine }\end{array}$ \\
\hline $\begin{array}{l}\text { Teneur moyenne des alinents } \\
\text { (2 fabrications) }\end{array}$ & 0 & $82-9 x$ & $15-25$ & $\begin{array}{l}36-38 \text { ()xytétracycline } \\
8-11 \text { Olćandomycine }\end{array}$ \\
\hline
\end{tabular}

(1) Non commercial du mélange ( $x y$ tétracyline + Oléandomycine dont les proportions de matière active sont de $4 / 1$.

\section{RÉSULTATS}

\section{Expérience 1. - Animaux alimentés à volonté}

Le poids moyen des animaux après 4 semaines varie dans les limites assez larges (fig. I). On constate en effet une amélioration du poids moyen à 9 semaines, de $3 \mathrm{~kg}$ dans le lot IV (taomycine) par rapport au lot I ('l'émoin). Lans l'ensemble (tabl. 2), les animaux recevant les aliments supplémentés ont des vitesses de croissance plus élevées (I I à 25 p. Ioo supérieures au témoin), mais cette amélioration n'est significative que pour les régimes contenant 1'oléandomycine et la " taomycine "; le lot supplémenté en oxytétracycline présente une stimulation de croissance plus faible et non significative.

On constate d'autre part, une augmentation significative de la quantité moyenne d'aliment consommée par porcelet dans tous les lots où la stimulation de croissance a été observée. En outre, une légère augmentation de l'efficacité alimentaire apparaît dans le lot supplémenté en oléandomycine.

L'état sanitaire a été excellent dans tous les lots.

(1) L'aliment de sevrage avait la composition suivante : p. I00: avoine décortiquée : $20 ; 13 l e ́$ : 16 ; Orge: ıo; Manioc : ro; Huile d'arachide : 5 ; Sucre dénaturé : Io; Tourteau de soja : I5; Farine de poisson :5; Lait écrémé sec : 5 ; Minéraux, Vitamines : 3,5 ; Mélange antibiotique : 0,5.

Annales de Zootechnie. - Ig68. 


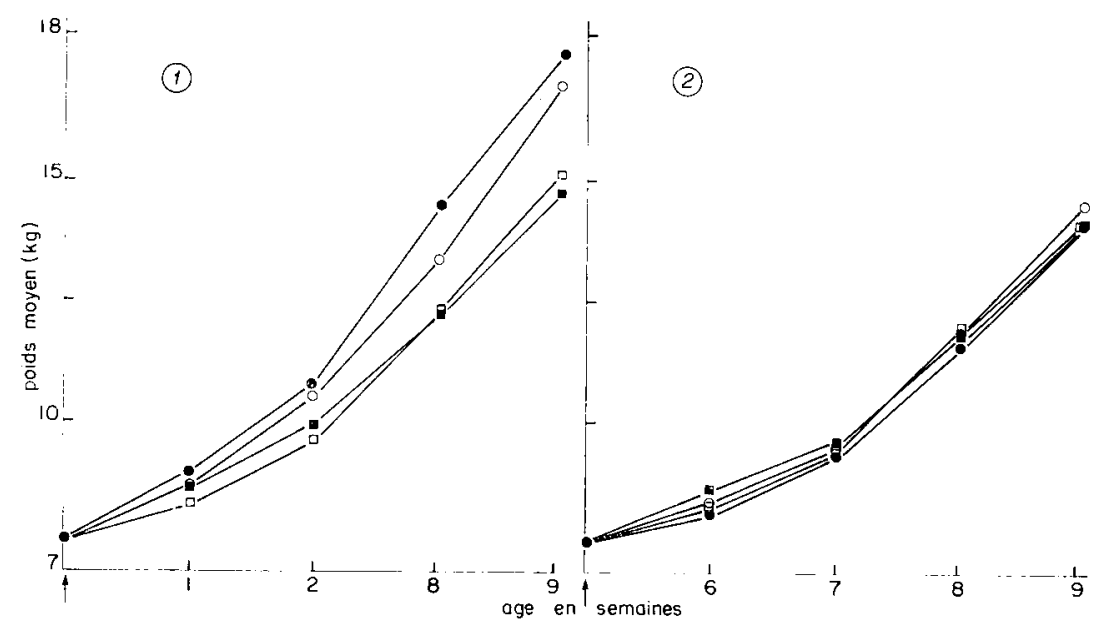

Fug. I. - Evolution die poils moyen des animaux pour les différcnls régimes

(I) animaux nourris à volonté (2) animaux nourris en quantité égale

- Lot témoin

:- - oxytctracycline

- - taomycine

-. oléandomycine

$\uparrow$ sevrage

TABLEAU 2

Performances de croissance des animaux alimentés à volonté en groupe (Expérience I)

\begin{tabular}{|c|c|c|c|c|}
\hline $\begin{array}{l}\text { Aliment supplémenté } \ldots \ldots \ldots \ldots \\
\text { (Nombre d'animaux) } \ldots \ldots \ldots \ldots \ldots\end{array}$ & $\begin{array}{l}\text { Témoin } \\
(16)\end{array}$ & $\begin{array}{l}\text { Oxytétracyline } \\
(16)\end{array}$ & $\begin{array}{l}\text { Oléandoinycine } \\
\qquad(16)\end{array}$ & $\begin{array}{c}\text { Taomycine } \\
(16)\end{array}$ \\
\hline Poids au sevrage, $(\mathrm{kg})$ (5 semaines) & 7,67 & 7,66 & 7,67 & 7,67 \\
\hline Gain cle poids $(\mathrm{g} / \mathrm{j})$ (5 à 9 semaines) & 273 & 302 & $353^{* *}$ & $369^{* *}$ \\
\hline (Valeur relative)............ & $(100)$ & (111) & $(\overline{129)}$ & (135) \\
\hline Quant. moy. d'alim. consom. (g/j) & 509 & 546 & $597 * *$ & $739 * *$ \\
\hline (Valeur relative)............. & $(100)$ & $(107)$ & $(117)$ & $(1 / 15)$ \\
\hline Efficacité alimentaire : & & & & \\
\hline $\begin{array}{l}\mathrm{kg} \text { de gain } / \mathrm{kg} \text { d'aliment } \ldots \ldots \ldots \ldots \\
\text { (Valeur relative) } \ldots \ldots \ldots \ldots \ldots \ldots\end{array}$ & $\begin{array}{l}0,5 / 4 \\
(100)^{2}\end{array}$ & $\begin{array}{l}0,55 \\
(103)^{-}\end{array}$ & $\begin{array}{l}0,59^{*} \\
(110)^{-}\end{array}$ & $\begin{array}{c}0,50 \\
(94)\end{array}$ \\
\hline
\end{tabular}

* $\mathrm{P}<0,05$. Seule cette valeur est significativement différente de la suivante.

** $\mathrm{P}<0,01$. Les valeurs réunies par un trait sont significativement différentes des autres. 


\section{Expérience $2-$ Animaux rationnés}

Les résultats moyens sont présentés à la figure $\mathrm{I}$ et au tableau 3.

Quel que soit le lot, on n'observe que de faibles variations du poids et de la vitesse de croissance des animaux. La supplémentation a un effet faible et non significatif sur la vitesse de croissance ( 3 à 9 p. IOo).

On observe toutefois une légère amélioration de l'efficacité alimentaire dans le lot recevant l'oléandomycine par rapport au régime témoin.

L'état sanitaire des animaux n'est que peu affecté par la supplémentation. On a noté des cas de diarrhée avec la fréquence suivante, pour toute la période :

$\begin{aligned} \text { I } & \text { Lot témoin } \\ \text { II } & \text { Lot Oxytétracyline } \\ \text { III } & \text { Lot Oléandomycine } \\ \text { IV } & \text { Lot Taomycine }\end{aligned}$

0,9 jour de diarrhée par porcelet

I, I jour de diarrhée par porcelet

I,o jour de diarrhée par porcelet

r,o jour de diarrhée par porcelet

les différences observées ne sont pas significatives.

TABIEAU 3

Performances de croissance des animaux alimentés et rationnés individuellement (Expérience 2)

\begin{tabular}{|c|c|c|c|c|}
\hline $\begin{array}{l}\text { Aliment supplémenté } \ldots \ldots \ldots \ldots \\
\text { (Nombre d'animaux) . . . } \ldots \ldots \ldots\end{array}$ & $\begin{array}{l}\text { 'T'émoin } \\
\text { (11) }\end{array}$ & $\begin{array}{l}\text { Oxytétracycline } \\
(11)\end{array}$ & $\begin{array}{l}\text { Oléandomycine } \\
\text { (11) }\end{array}$ & $\begin{array}{l}\text { Taomycine } \\
\text { (11) }\end{array}$ \\
\hline Poids départ $(\mathrm{kg})$ (5 semaines)... & $7,6 \mathbf{t}^{\prime}$ & 7,63 & 7,67 & 7,66 \\
\hline $\begin{array}{l}\text { Gain de poids } g / j \text { (5 a } 9 \text { semaines)... } \\
\text { (Valeur relative }) \ldots \ldots \ldots \ldots \ldots \ldots\end{array}$ & $\begin{array}{c}231 \\
(100)\end{array}$ & $\begin{array}{c}239 \\
\left(10^{\prime}\right)\end{array}$ & $\begin{array}{c}257 \\
(109)\end{array}$ & $\begin{array}{c}244 \\
(105)\end{array}$ \\
\hline $\begin{array}{l}\text { Quantité d'alim. consommée/j. (g) } \\
\text { (Valeur relative)............. }\end{array}$ & $\begin{array}{c}423 \\
(100)\end{array}$ & $\begin{array}{l}433 \\
(102)\end{array}$ & $\begin{array}{c}433 \\
(102)\end{array}$ & $\begin{array}{c}424 \\
(100)\end{array}$ \\
\hline $\begin{array}{l}\qquad \text { Efficacité alimentaire }: \\
\mathrm{kg} \text { de gain } / \mathrm{kg} \text { d'aliment } \ldots \ldots \ldots \\
\text { (Valeur relative) } \ldots \ldots \ldots \ldots \ldots \ldots\end{array}$ & $\begin{array}{c}0,516 \\
(100)^{-1}\end{array}$ & $\begin{array}{c}0,552 \\
(101)^{-2}\end{array}$ & $\begin{array}{l}0,593^{*} \\
(109)\end{array}$ & $(105)^{0,575}$ \\
\hline
\end{tabular}

$*<0,05$. Valeur significativement supérieure au témoin.

\section{DISCUSSION}

Deux faits apparaissent dans les résultats des deux expériences :

Io il existe des différences de stimulation de la croissance du porcelet sevré à 5 semaines suivant les antibiotiques et dans les mêmes conditions d'élevage et de milieu. 
L'oléandomycine incorporée à 20 p.p.m. semble stimuler fortement la croissance ( + 29 p. roo) alors que 1'oxytétracycline utilisée à Ioo p.p.m. dans notre élevage depuis plus de 5 ans, n'agit que faiblement.

Les effets de stimulation trouvés pour l'oléandomycine seule sont comparables à ceux trouvés par HAWBAKER et al. (I960) ; LLoYd et al. (I96r), et par SALMONLEGAGNEUR et MrcheI, (I955 et I96I) pour la spiramycine. Ils sont nettement plus élevés que les valeurs trouvées par d'autres auteurs sur le Porc en croissance ou âgé de plus de deux mois (Smith et al., I963; Puaci et al., I965; Krígir et Borman, I964).

L'oxytétracycline seule même à dose élevée (Ioo p.p.m.), n'a pas un effet aussi spectaculaire; ceci est en accord avec les résultats de KRÜGER et BoRMAN, I964. D'autre part il semble que la stimulation de croissance observée ait varié avec le temps : elle a baissé dans notre propre élevage de plus de $5^{\circ} \mathrm{p}$. Ioo depuis 4 ans (JoUANDET et al., I964).

Par contre, on peut souligner que 1'oxytétracycline agit encore de façon très importante si on lui adjoint l'oléandomycine et nos résultats nous autorisent à confirmer l'effet de synergie des deux antibiotiques. Cet effet, signalé par FERranino et $a l$. (I960) a été également observé par Müxchberg et al. (I963), par Isakov (I963), KRÜGER (I g64), avec des stimulations moins importantes sur le Porc après 2 mois. Ėnfin, la propriété de synergie de l'oxytétracycline employée en association et mise en évidence in vitro par une augmentation très nette du pouvoir bactéricide (Russis,, I962) a déjà été signalée in vivo chez le Porc lorsqu'elle est associée à la pénicilline seule (BECKER et al., I953) ou à la pénicilline et à un sulfamide (RAYNAUI), I967).

L'influence généralement faible de la supplémentation en antibiotique sur l'efficacité alimentaire ou sur la diarrhée des animaux se retrouve quel que soit le mode d'alimentation. Ces résultats sont en accord en particulier avec ceux de Jounardet et al. (I964), et de VLRxox et al. (I962) pour l'oléandomycine.

$2^{\circ}$ Parmi les nombreux mécanismes d'action des antibiotiques (FrançoIs, I962), nos résultats soulignent que la supplémentation agit par l'intermédiaire de l'appétit et peut-être de l'amélioration de l'état sanitaire des porcelets au moment du sevrage.

En effet, dans l'expérience 2, lorsque les animaux supplémentés sont alimentés en quantité égale à celle des animaux témoin, l'effet de stimulation de la croissance paraît limité, en accord avec les résultats obtenus chez le porcelet par JoUANDET et al. (I964).

Reçu pour publication en fírier 1968.

\section{REMERCIEMENTS}

A la Société industrielle de Biochimie pour nous avoir fourni les antibiotiques nécessaires à l'expérience et avoir réalisé les dosages de contrôle des quantités incorporées aux aliments (Dr J.-P. Raynaud). 


\title{
SUMMARY
}

\author{
SUPPLEMENTATION OF EARLY-WEANING DIETS FOR PIGLETS WITH ANTIBIOTICS \\ (OXYTETRACYCLINE, OLEANDOMYCIN AND A COMBINATION OF THEM)
}

The effect of supplements of antibiotics (oxytetracycline roo p.p.m. ; oleandomycin 20 p.p.m. to early-weaning diets for piglets was studied by comparing the effect of the antibiotics alone or combined (Taomyxin : 40 p.p.m. oxytetracycline + ro p.p.m. oleandomycin). In the first experiment effect on growth and efficiency of feed utilization of the diets was estimated in 64 piglets fed to appetite in 4 groups, group I control, group II oxytetracycline, group III oleandomycin and group IV Taomyxin. 'The second experiment was with 44 selected piglets from I I litters pair-fed for 4 weeks from weaning at 35 days of age.

Growth was 29 per cent greater than in controls with oleandomycin and 35 per cent greater with Taomyxin only when the piglets were fed to appetite. Oxytetracycline alone had no effect. When the piglets were pair-fed there was non increase in growth rate. Efficiency of feed utilization was little improved in general, and conclusions are drawn concerning the mode of action of antibiotics. Supplementation of weaning diets greatly increases appetite of the animals andimproves their state of health which accounts for a great part of the improvement of growth which was recorded.

\section{RÉFÉRENCES BIBLIOGRAPHIQUES}

Becker D. E., Terrill S. W., Meade R. J., Euwards R. M., i952. The efficiency of various antibacterial agents for stimulating the rate of gain in the pig. Antibiolics Chemolher., $2,42 \mathrm{I}-425$.

Becker D. E., Terrill S. W., Lassister J. W., Nelson T. S., Gard D. I., i953. The influence of antibiotic combinations on the growth response of the pig. Antibiotics Chemother., 3, $77^{8-782 .}$

Ferrando R., 'Tieodossiades G., ig6o. The action of oleandomycin alone or mixed with terramycin on the growth rate of pigs. Proc. Pfizer Luropean Agric. Res. Conf. Lucerne.

Françors A.-C., 1962. Wode of action of antibiotics on growth. Wha. Rev. Nulr. Diel, 3, 2 I-64.

Hawbaker J. A., Diaz F., Speer V.C., Hays V. W., Catron D. V., ig6o. The effect of oleandomycin on the performance of the young growing pig. J. Anim. Sci., 19, 800-802.

Isakov D., rg63. Comparative value of tamycin and terramycin in the fattening of young swine. Vet. Glasn., 17, $239^{-246 .}$

Jouandet C., Aumatre A., Shlmon-Legagneur E., ig64. Influence des antibiotiques sur la croissance des porcelets sevrés à 5 semaines. Ann. Zootech., 13, II.-S. I I 3 -127.

Krüger L., BormanN P., I964. Über die Wirkung von Támycin, Terramycin und Oleandomycin "Pfizer" bei Aufzucht und Mast schweinen. Z. Tierphysiol. T'ierernähr. Futtermittelk., 19, 310-3I6.

Lloyd I. F., Crampton F. W., Mowat D. N., ig6r. Effect of calcium : phosphorus ratio, oleandomycin and protein level on the performance of early-weaned pigs. J. Anm. Sci., 20, $176-179$.

Magruder D. A., Sherman W. C., Revnold W. M., Lutiler H. G., i958. A new antibiotic, oleandomycin, in swine rations. J. Anim. Sci., 17, II 77 .

Münciberg F. Von., Halama A. K., I 963 . Taomycin in der Fütterungr von Absetzferkeln. Z. Tierphysiol. Tierernähr. Fultermittelk., 18, 242-250.

Puach V., Pop-Cenic S., Vuckovic M., ig65. Oleandomycin in the rations of fattening swine. Vet. Glas., 19, $773-778$.

Raynaud J.-P., I967. Aliments thérapeutiques. Expérimentation d'un mélange de deux antibiotiques et d'un sulfamide dans la prophylaxie des maladies du sevrage du porcelet. Rec. Med. Vet. ., 143, 66r-675.

Rérat A., Jouandet C., Lougnon J., r965. Influence des antibiotiques sur la rétention azotée chez le Rat en croissance. Ann. Biol. anim. Biochim. Biophys., 5, 4I-6I.

Russel A. D., I962. Antibiotics. IV. Antibiotic synergism and antagonism. Lab. Pract., 11, 840-84I.

Salmon-Legngnetr E., Michel M., 1955. Action des antibiotiques sur porcelets allaités. Ann. Zootech., 4, I $53^{-I} 55$.

Salmon-Legagneur E., i $96 \mathrm{r}$. Les antibiotiques et la croissance du Porc. IX. Action de la spiramycine sur la croissance du porcelet avant et après le sevrage. Ann. Zootech., 10, 39-43.

Smith W. C., Adam J. I., Tonks H. M., I963. The supplementation of pig diets with oleandomycin. Anim. Prod., 5, 20I-208.

Vernon J., Mercer E. A., Rosen G. D., I962. The effects on production efficiency of oleandomycin and oxytetracyclin fed at two levels in the diet of heavy pigs. Anim. Prod., 4, 279. 